\title{
Alcohol intoxication progressively impairs drivers' capacity to detect important environmental stimuli.
}

Authors:

Martin Henry Plawecki, M.D., Ph.D. ${ }^{a 1}$

Sarah Koskie, Ph.D. ${ }^{b}$

Ann Kosobud, Ph.D. ${ }^{\text {a2 }}$

Michael D. Justiss, Ph.D., OTR/L ${ }^{\mathrm{b}, \mathrm{c}}$

Sean O'Connor, M.D. ${ }^{\text {a1 }}$

a: Indiana University School of Medicine, Indianapolis, IN

Departments of: a1 Psychiatry, a2 Neurology

b: Indiana University Purdue University Indianapolis,

Dept. Electrical and Computer Engineering

c: Indiana University School of Health and Rehabilitation Sciences, Dept. of Occupational Therapy

Corresponding Author:

Sean O'Connor, M.D.

Professor of Psychiatry and Biomedical Engineering Indiana University School of Medicine and Purdue University UH 5510 - General Clinical Research Center 500 University Boulevard Indianapolis, IN, 46202 USA

(317) 989-4186 oconnor1@iu.edu

This is the author's manuscript of the article published in final edited form as:

Plawecki, M. H., Koskie, S., Kosobud, A., Justiss, M. D., \& O'Connor, S. (2018). Alcohol intoxication progressively impairs drivers' capacity to detect important environmental stimuli. Pharmacology Biochemistry and Behavior. https://doi.org/10.1016/j.pbb.2018.05.009 


\section{$\underline{\text { Abstract }}$}

Rationale: Alcohol intoxication impairs driving skills, leading to an increased frequency of accidents and crash fatalities. Inebriation may specifically impair environmental vigilance, reducing the driver's capacity for attention to stimuli that are relevant to successful navigation.

Objectives: We examined the separate and interactive effects of breath alcohol concentration $(\mathrm{BrAC})$ and simulated driving scenario on the capacity to correctly identify visual stimuli embedded in the environment.

Methods: Ten healthy young adult drivers (6 males; 4 females) each performed 4 driving scenarios at each of 3 steady breath alcohol concentration levels $(0,60$ and $100 \mathrm{mg} / \mathrm{dl})$. Scenarios were based on speed or distance keeping while navigating a rural 2-lane road in daytime or nighttime conditions. Drivers pressed a button on the steering wheel corresponding to the direction of an arrow (up or down) which appeared briefly on road signs embedded in the environment, either overhead or on the roadside.

Results: Increasing level of $\mathrm{BrAC}$ and subjective scenario difficulty manifested significant, separate, but not interactive influences in association with the number of arrows correctly identified. Significant impairments could be detected at a level of BrAC below the current American limit for legal operation of a motor vehicle.

Conclusions: Environmental vigilance is subject to impairment by either/both alcohol intoxication and driving conditions.

Keywords: Simulated driving; scenario difficulty; alcohol; vigilance; clamping. 
Introduction:

Environmental vigilance, defined as the ability to detect and interpret environmental cues relevant to driving, is important to safety while operating a motor vehicle. Visual cues such as posted road signs prompt attention to speed, curves, exits or crossings, potential for bridge icing, road work, one-way traffic and other environmental factors that impact safety. In addition to these fixed aspects of roadway infrastructure, there are other more variable environmental factors that influence driving behavior and safety: weather, pot-holes, pedestrians, cyclists, other vehicles and wildlife. Behavioral responses are required to adjust to these unforeseen circumstances, requiring focus, alertness, and preparedness for maintaining safe navigation. Missing or misinterpreting such cues may increase the crash risk.

Alcohol intoxication impairs driving skills. The use of a high-level driving simulator for such studies seems likely to underestimate the effects of alcohol and drugs on driving performance (Veldstra et al, 2015; Kenntner-Mabiala et al, 2015). However, the association of intoxication and impairment in simulator performance is well-documented (e.g. Laude and Fillmore, 2015, Bernosky-Smith et al, 2011), thus was not the object of our experiment. Our goal was to assess the effect of alcohol intoxication on the capacity for environmental vigilance during simulated driving.

Distraction also impairs driving skills by dividing the driver's attention. Texting, conversing, eating, grooming, and adjusting dashboard controls are examples of common distractions that have been shown to reduce a driver's performance at lane-keeping, distance keeping, speed control and other conventional measures of driving skills. The brain activity required to perform familiar autonomous motor skills (such as driving a familiar route) differs from the neural activity required to recognize distractions (Schweizer et al, 2013, Meda et al, 2009). Attention can be

Acronyms employed: BrAC:end-expiratory breath alcohol concentration (mg/dl), TASI:Transportation Safety Institute, CAIS:Computer-assisted Alcohol Infusion System, PBPK:Physiologcally-based Pharmacokinetic model, DA:Daytime Autonomous; one of four driving scenarios employed, NA:Nighttime Autonomous; NDK:Nighttime Distance Keeping; NDKH:NDK aided by an HMI: Human Machine Interface, CRC:Clinical Research Center, UDA task:Up/Down Arrow probe of environmental vigilance. 
provided to both driving and distraction simultaneously, but not without sacrifice of driving performance (Strayer and Johnston, 2001). Alcohol intoxication, even below the current per se legal limit, appears to dramatically increase the impact of distractions on driving performance (Van Dyke and Fillmore, 2015, Rakauskas et al, 2008). That influence is one reason that NHTSA proposes to lower the American limit to the European standard of $50 \mathrm{mg} / \mathrm{dl}$ (NTSB, 2013, Fell and Voas, 2014).

We considered 3 hypotheses: that alcohol intoxication would impair environmental vigilance, that significant impairment could be measured at levels of intoxication below the current per se limit for operating a motor vehicle in the USA, and that the degree of impairment would increase with the BrAC level. We conducted a within-subject study of the effect of constant BrACs, compared to sobriety, on scores on a novel vigilance task, conducted during each of 4 driving scenarios in a driving simulator.

\section{Methods:}

Participants: Recruiting sought young, physically and emotionally healthy participants who had completed previous alcohol infusion studies and who had expressed interest in participating in additional projects. A Time-Line Followback (Sobell and Sobell, 1992) interview documented reported drinking history for the previous 35-days. Exclusion criteria included current psychoactive medication, pregnancy, history of any drug dependence, and illicit substances in the urine on the day of testing. Subjects were requested to avoid alcohol for 24 hours before testing.

Apparatus and materials: The Transportation Active Safety Institute (TASI) driving simulator. TASI is a Signature Center research enterprise of Indiana University Purdue University Indianapolis. TASI comprises a test track, manufacturing and instrumentation facilities, accident and driving database analytic resources, ten faculty members and a DS-600c Driving Simulator 
http://www.tasi.iupui.edu/facilities/driving-simulator-laboratory/. The TASI simulator comprises the front passenger cabin of a Ford Focus including the driver/passenger seats and all driver controls, a 3-segment environment display, comprising $6 \times 10 \mathrm{ft}$. video projections, arranged as a segmented circle covering 210 degrees driver's field of view. A high-definition rendering of the automobile's changing environment is refreshed at $60 \mathrm{frame} / \mathrm{sec}$. The vehicle's 3 rear-view mirrors are also video monitors reflecting the appropriate image from the driver's vantage. The vehicle is equipped with $a+/-5$ degree pitch actuator that approximates the effect of braking and acceleration. All vehicle controls are instrumented and serve as real-time inputs to the vehicle/environmental system. Input signals measuring driver behaviors on related tasks can be added. All current inputs, 4-axis environmental position, 3-axis vehicle accelerations, speeds, lane position, and environmental views are recoded every $16.7 \mathrm{msec}$.

Administration of Alcohol; the Computer-Assisted Alcohol Infusion System (CAIS): Our lab invented CAIS and employed it in this project. CAIS is a proprietary set of software, hardware and technician interfaces for precise control of human brain exposure to alcohol. CAIS uses a physiologically-based pharmacokinetic (PBPK) model for alcohol (Ramchandani et al, 1999) with an intravenous infusion rate of $6 \%$ alcohol in half-normal saline as the input and $\mathrm{BrAC}$ as the primary output. BrAC is a good proxy for brain exposure to alcohol (Jones et al., 1997, Gomez et al., 2012). CAIS utilizes a proportional controller on the instantaneous error between the model-predicted BrAC and the desired BrAC to drive the alcohol infusion rate. Thus, exercising the model computes the infusion rate profile required to achieve the desired $\mathrm{BrAC}$ and thus brain alcohol exposure. CAIS then administers the profile by control of a dual infusion pump delivering $6 \%$ alcohol in half-normal saline to a vein in the inside of the elbow of the driver's non-dominant arm. Since the PBPK model parameters can be specified for each individual (Plawecki et al., 2007), all subjects are exposed to the same BrAC trajectory even though the required infusion rate profiles differ. 


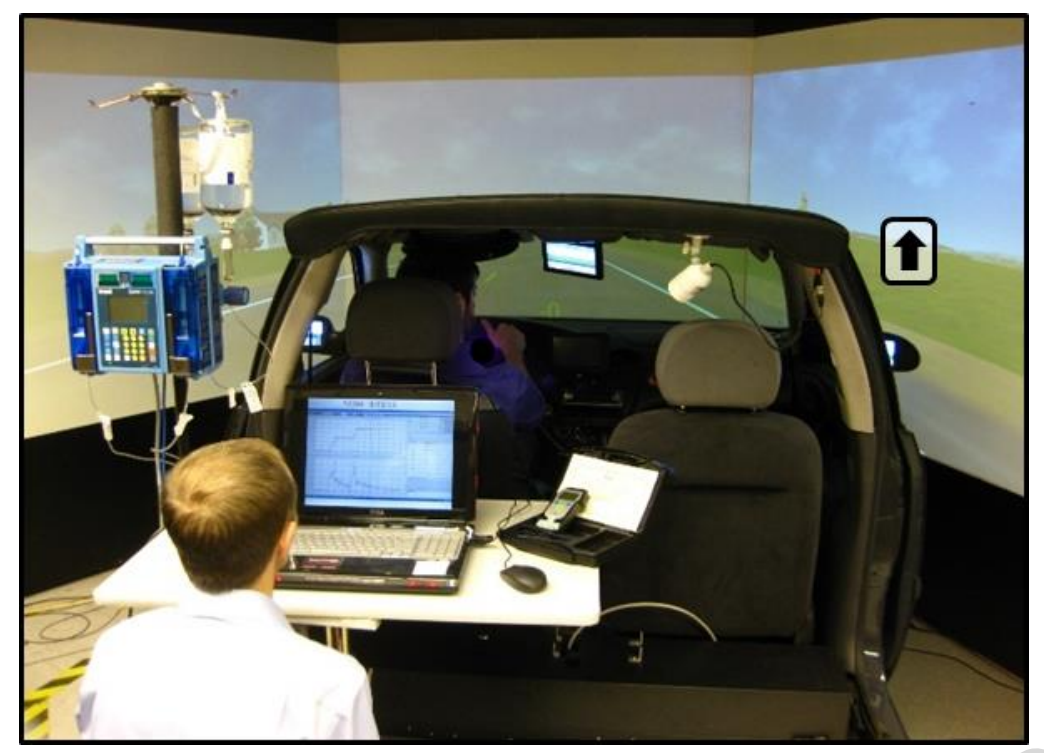

Figure 1: Apparatus employed in this study. The TASI DS-600c Driving Simulator is shown from the rear along with the CAIS apparatus (alcohol infusate, computer-controlled infusion pump, laptop with PBPK model embedded in CAIS software and breath alcohol concentration meter. The driver's thumb is pushing a button mounted on the steering wheel to indicate the perceived direction of the arrow on the pseudo-road sign moving past him with the environment.

The stepped BrAC Clamp: CAIS BrAC Clamping raises the subject's BrAC at a specified rate to a prescribed level and maintains that level indefinitely, the alcohol "clamp"; we routinely perform studies using BrAC clamps lasting up to 3 hours (Ramchandani and O'Connor, 2006, Plawecki et al., 2008). For this project, we modified the paradigm to implement clamps at successively higher BrAC. Subjects' BrACs were clamped for $45 \mathrm{~min}$ at each at 0 (no alcohol), 60 and 100 $\mathrm{mg} / \mathrm{dl}$.

Up/Down Arrow (UDA) task: We developed the UDA task as a quantifiable, environmentally valid test of vigilance. At intervals uniformly distributed in the [7-13] sec range, 35 trials were presented during every driving scenario tested (below). In each trial, a symbol mimicking a 'oneway' road sign (but with vertical orientation and without text, Figure 1) appeared for $1.5 \mathrm{sec}$, dissolving at the beginning and end of its appearance. The signs traveled with the environment, appearing at any of 3 randomly assigned positions relative to the roadway centerline: 0 (overhead at $15 \mathrm{ft}$.) or \pm 30 degrees visual angle (at $8 \mathrm{ft}$. above road elevation and offset $10 \mathrm{ft}$. from outside lane border). Half the symbols displayed an arrow pointing up; half 
pointing down; the direction sequence pseudo-randomly programmed and changing with each scenario. The driver's steering wheel was equipped with 3 buttons, each operated with ease by the right thumb. The top button was labeled 'Up' and had an elevation in its center; the bottom button: 'Down' with a depression in the center; and the center button: 'Pass' (I saw it, but I couldn't tell if it was up or down) with a smooth surface. The driver's instructions were to press the appropriate button each time an arrow sign appeared. Vigilance was quantified as the number of arrows correctly identified.

Driving Scenarios: In each of the following scenarios, the $5.0 \mathrm{Km}$-long course comprised a 30 ft. wide 2-lane asphalt road, winding through gently rolling hills with farms to either side and with both oncoming and passing traffic imbedded. A few, one-time environmental distractions were also programmed (e.g. a deer on the side of the road, a recent auto accident attended by police car and ambulance). One $0.5 \mathrm{~km}$ section had some sharp curves, but the overall course was easy to navigate. Posted speed limits varied between 35 and $55 \mathrm{mph}$ and changed 4 times during the scenario. The simulation began with the car stopped and ended after the subject brought the car to a full stop at the only stop sign on the course. Each scenario took between 5.5 and 6.5 minutes to navigate.

Daytime Autonomous (DA) scenario: The subject was instructed to drive safely at the posted speed limit in bright daylight, dry-road conditions and to press the correct button for each up/down arrow observed. The rationale was to demonstrate that effects of alcohol on vigilance should be observable on a relatively easy driving task.

Nighttime Autonomous (NA) scenario: The same course and instructions as DA were employed, but driven in the opposite direction. A no-moon, but otherwise clear nighttime environment was simulated, and the subject had full control of the vehicle headlights. The 
rationale was to explore a potential interaction between the effects of lighting conditions and alcohol on vigilance.

Nighttime Distance Keeping (NDK) scenario: All conditions of the NA scenario were preserved, but an additional vehicle (a white SUV) was inserted in the scenario, and the instructions were changed from 'follow the posted speed limit' to 'follow the lead vehicle at a constant distance' (nominally 35 meters). The lead vehicle accelerated and decelerated at $0.5 \mathrm{~m} / \mathrm{sec}^{2}$, with smoothing to constant velocity in order to track the pre-programmed speed-limit profile, and swerved around the embedded accident scene to avoid collision. It also ignored the stop sign that, when recognized by the driver, terminated each scenario. Nighttime Distance Keeping with HMI support (NDKH) scenario: This scenario added an Human-Machine Interface (HMI) display to the NDK setup driven in the opposite direction. The simulator software projected an opaque, solid color-coded rectangular visual display ( $3 \times 2$ degrees visual field) onto the video screen directly in front to the driver at hood level. The display appeared only when the distance between the lead and subject vehicle was too small (red; $<25 \mathrm{~m}$ ) or too great (blue; $>45 \mathrm{~m}$ ). The rationale for including the NDKH scenario was to gather preliminary data for eventual testing of the hypothesis: while human-machine interfaces may improve sober driving performance, their use may interact with alcohol intoxication to degrade driving performance to levels below the no-HMl condition.

\section{Procedures:}

Subject participation occurred on a single day of testing. A subject arrived on the Indiana University School of Medicine Clinical Research Center (CRC) at 9 am. After providing a BrAC measurement of $0 \mathrm{mg} / \mathrm{dL}$, the subject furnished informed consent for the study, ate a standardized 350 calorie breakfast, supplied a urine sample for testing for illicit drugs (and hcG 
for pregnancy in females) and performed a 35-day Time-Line Follow-Back history of recent drinking. A nurse placed a heparinized 20 gauge indwelling catheter in an ante-cubital vein of 1 arm and the subject was escorted to the TASI Driving Simulator Lab, walking for about 10 min. At TASI, we familiarized the subject with the simulator and the tasks to be performed, and the subject practiced each driving scenario including the UDA task until $s /$ he felt comfortable with the experience. At $\sim 11$ am, we connected the subject's to the Y-connected outputs of 2 IMedPCTx pumps (1998 $\mathrm{ml} / \mathrm{hr}$ infusion capacity), each fed by 1 liter of $6.0 \%(\mathrm{v} / \mathrm{v})$ ethanol in halfnormal saline. The technician entered the subject's age, height, weight and gender into CAIS which converted the information to 5 physiologic parameters employed by the now subjectspecific PBPK model of alcohol distribution and elimination (Plawecki et al., 2007).

Each subject performed all 4 driving scenarios at each level of clamped $\mathrm{BrAC}$; the order within level was counter-balanced in a pseudo-randomized fashion. When sober testing (0 $\mathrm{mg} / \mathrm{dll}$ ) was completed, we disconnected the pumps and asked the subject to void his/her bladder. When reconnected, CAIS raised the subject's BrAC at $4.0 \mathrm{mg} / \mathrm{d} / \mathrm{min}$, then held it steady at $60 \mathrm{mg} / \mathrm{dl}$ for $45 \mathrm{~min}$. After another bath-break, CAlS raised the BrAC at the same rate of increase and held it at $100 \mathrm{mg} / \mathrm{dl}$ until testing was completed. The subject performed driving scenarios only during intervals of constant BrAC. After completion of the last scenario at $100 \mathrm{mg} / \mathrm{dl}$, we disconnected the subject's infusion line from the pumps. The subject relaxed on a couch in the Simulator Lab for an hour, using that time to begin recovery, and then provided answers to a structured debriefing questionnaire about his/her experience. We then escorted the subject, riding in a wheelchair, back to the $\mathrm{CRC}$, where s/he continued recovery in a private room. We provided a lunch and tracked BrAC measurements throughout recovery to $20 \mathrm{mg} / \mathrm{dl}$. Before discharge from the CRC, we offered the subject an evening meal, paid him/her $\$ 100$ in cash plus a parking voucher and returned his/her car keys. The typical discharge time was $\sim 6 \mathrm{pm}$. 
Data Collection: Relevant variables, sampled and recorded every $16.7 \mathrm{msec}$ throughout each scenario by the driving simulator software were: System Time, Video Frame Number, Vehicle Speed, Lane Position, (Speed Limit - Speed), Steering Angle, Braking \%, Engine RPM, and Lateral and Longitudinal Acceleration. In addition: Distance to the Lead vehicle, Lead Vehicle Speed and Lead Vehicle Longitudinal acceleration were sampled and recorded during the NDK and NDKH scenarios. These data were used to confirm an alcohol effect on driving performance, but did not comprise dependent measures, per se.

We recorded several features for each UDA arrow stimulus: frame number and longitudinal position of onset, horizontal visual angle relative to roadway centerline when in view, the arrow's direction (pointing up or down), and the driver's response to the arrow (Up, Down, Pass (or none $=$ miss $)$ ).

Data reduction: We wrote Excel macros to convert the recorded data to the analytical database, comprising single-number scores for the following variables in each of the 120 subject/scenario/BrAC combinations:

UDA Task: \# Correct Responses

Vehicle Longitudinal Acceleration: mean (abs value), SD (value)

Controls: (Steering Wheel Angle, Braking \% when engaged, Engine RPM); SD (value)

Speed: Sum over scenario time of (speed limit - vehicle speed $)^{2}$ when speed $>25 \mathrm{mph}$ Vehicle Lateral Acceleration, $\mathrm{m} / \mathrm{sec}^{2}$, straight segments: mean (abs value), SD (value) Vehicle Lateral Acceleration, $\mathrm{m} / \mathrm{sec}^{2}$, curved segments: mean (abs value), SD (value)

\section{Statistical Analyses}

In order to confirm that alcohol exposure exerted a significant influence on driving performance, we examined lateral and longitudinal measures in the easiest (DA) scenario. We 
employed a repeated measures general linear model with simple contrasts using data from the DA scenario only, with BrAC was the repeated measure. Outcome measures comprised fraction of driving time spent braking, and the standard deviation of engine RPM, lane position, longitudinal acceleration, and lateral acceleration on curved segments of the scenario.

To confirm that the Scenario conditions varied in difficulty, we employed a repeated measures general linear model with simple contrasts using data from the $\mathrm{BrAC}=0 \mathrm{mg} / \mathrm{dL}$ condition only, with Scenario as the repeated measure. In order to test the hypothesis that environmental vigilance is sensitive to $\mathrm{BrAC}$, we assessed UDA performance using a multivariate general linear model, with both Scenario and BrAC as repeated measures. Tests of simple contrasts used $\mathrm{BrAC}=0 \mathrm{mg} / \mathrm{dL}$ and Scenario $=\mathrm{DA}$ as references.

\section{$\underline{\text { Results }}$}

Subjects: Ten subjects (4 Female, 1 African American), all physically healthy, young adults (aged 23-29) participated in the study. The subjects averaged 15.3 years of education and were all employed at time of testing; none had experienced any arrests for alcohol-related driving. As a group, they drank typically, if immoderately: reporting a (mean \pm sem) of $55.8 \pm 9.8$ European Standard Drinks, with 14.5 \pm 1.5 Drinking Days, in the previous 35 days; $4.0 \pm 0.6$ Drinks per Drinking Day. None had a history of legal troubles associated with drinking.

Nausea and adverse events: Three subjects reported transient mild nausea while they practiced the driving scenarios without any alcohol (Brooks et al, 2010), but none reported any nausea once testing began. No subject reported feeling fatigued by their experience in the simulator. There were no adverse events associated with this study which was approved by the Indiana University School of Medicine Institutional Review Board and reviewed by the IUSM Alcohol Studies Data Safety Monitoring Board. 
Stepped Clamps: The (mean \pm sem) modeled BrAC during segments when the BrAC was held steady at targets of 60 and $100 \mathrm{mg} / \mathrm{dl}$ were $60.2 \pm 0.8$ and $101.4 \pm 0.9 \mathrm{mg} / \mathrm{dl}$ respectively. The calculations are based on the continuous PBPK model output, verified by 4 or 5 actual BrAC measurements starting 5 min after the beginning of each clamped segment. The BrAC clamping performance was comparable to that evident in many other CAIS projects where the subject is seated quietly in a lab performing tasks that require no arm motion.

Driving performance as a function of scenario at $B r A C=0 \mathrm{mg} / \mathrm{dL}$ : During the debriefing interview, all subjects subjectively rated the difficulty of the driving scenarios in the same order: $\mathrm{NDKH}>\mathrm{NDK}>\mathrm{NA}>\mathrm{DA}$ at all three BrAC levels. They reported more perceived effort for both lateral and longitudinal control in association with nighttime than with daylight driving, and with distance-keeping than autonomous driving. We did not perform formal analyses of the relationships between reported subjective effort and driving performance across scenarios at any $\mathrm{BrAC}$. Figure 2 presents two objective measures when driving in the sober condition to illustrate the differences.
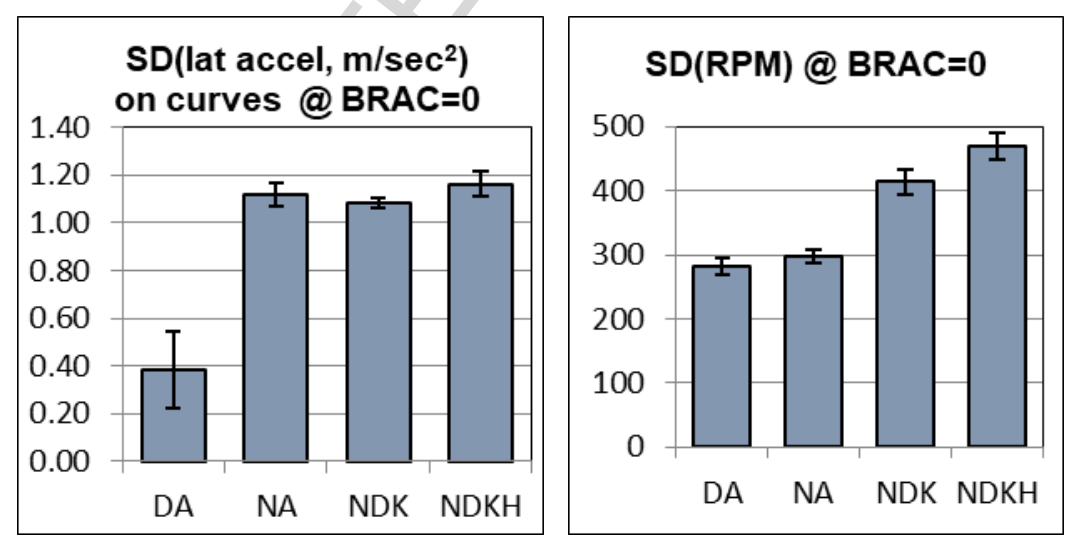

Figure 2: Lateral (left panel) and longitudinal (right panel) driving performance measures (mean \pm sem) of the effects of scenario in the sober state. The degraded performance reflects the subjects' reported perceptions of the order of scenario difficulty which was the same with and without alcohol. 
Driving performance as a function of BrAC level in the DA scenario: Subjects reported a progressive exposure-related subjective effect of alcohol ('intoxicated') in all driving scenarios, with greatest confidence in compensating for those effects in the Daytime Autonomous scenario. Nonetheless, statistical analysis revealed significant exposure-related effects of alcohol on driving performance (e.g. Figure 3).
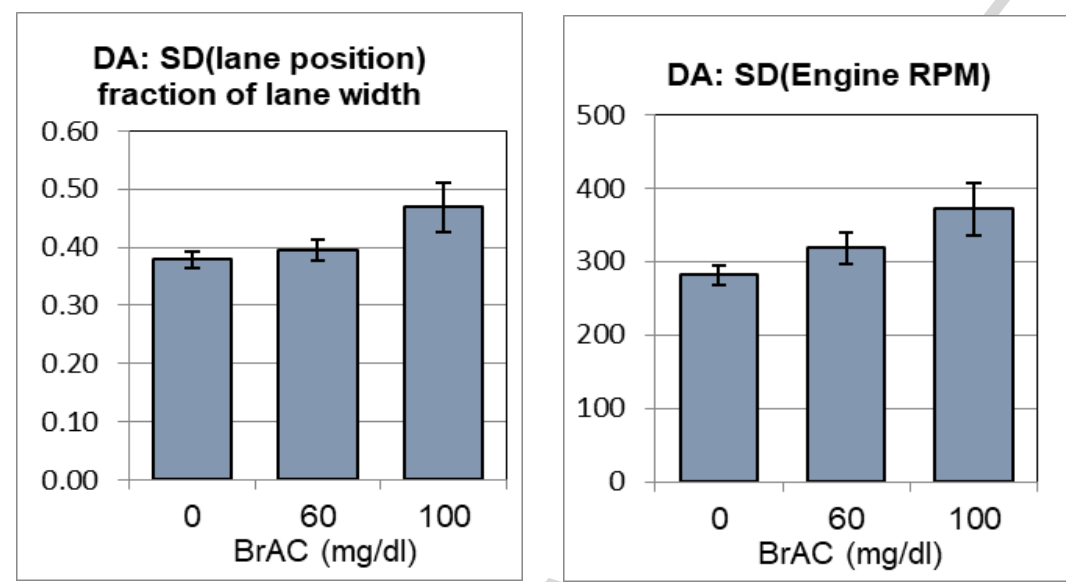

Figure 3: Lateral (left panel) and longitudinal (right panel) driving performance measures (mean \pm sem) of the effects of alcohol intoxication in the easiest (Daytime Autonomous, DA) driving scenario.

Table I documents notable BrAC level contrasts in the longitudinal and lateral axes on the driving scenario rated the easiest by all subjects.

Table I Exposure-related effects of alcohol on driving performance in the DA scenario

\begin{tabular}{|c|c|c|c|}
\hline Driving performance variable & BrAC level contrasts & F statistics & $\rho<$ values \\
\hline Longitudinal axis & $(\mathrm{mg} / \mathrm{dl}$; order noted $)$ & & \\
\hline Std. Dev. of acceleration & $0>60,100>0$ & $8.23,6.96$ & $0.03,0.02$ \\
\hline Engine RPM & $100>0$ & 8.85 & 0.02 \\
\hline Time spent braking & $60>0,100>0$ & $11.4,5.34$ & $0.008,0.05$ \\
\hline Lateral axis & & & \\
\hline Std. Dev. of acceleration & none & & $>0.10$ \\
\hline Std. Dev. of Lane position & $60>0,100>0$ & $5.13,7.9$ & $0.05,0.02$ \\
\hline
\end{tabular}


UDA Task Performance by driving scenario and BrAC: The principal hypotheses of this study were based on environmental vigilance, as quantified by the UDA task. Figure 4 shows that the number of arrows correctly identified varied by both scenario and $\mathrm{BrAC}$ level, decreasing with increasing $\mathrm{BrAC}$ level and in the inverse order of subjectively rated scenario difficulty. No subject reported the performance of the UDA task as a distraction to safe driving in any of the scenarios. BrAC emerged as an independent determinant of UDA task performance across scenarios for BrAC $60 \mathrm{mg} / \mathrm{dl}(\mathrm{F}=6.48, \rho<.05)$, and $\mathrm{BrAC} 100 \mathrm{mg} / \mathrm{dl}(\mathrm{F}=434 ., \rho<.001)$.

Scenario emerged as another independent determinant across BrAC levels: NA $(F=13.7, \rho<$ $.01)$; NDK $(F=25.5, \rho<.001)$ and NDKH $(F=55.9, \rho<.001)$. Testing for an interaction between BrAC level and Scenario did not yield statistical significance (Cohen's $d=0.12$ ).

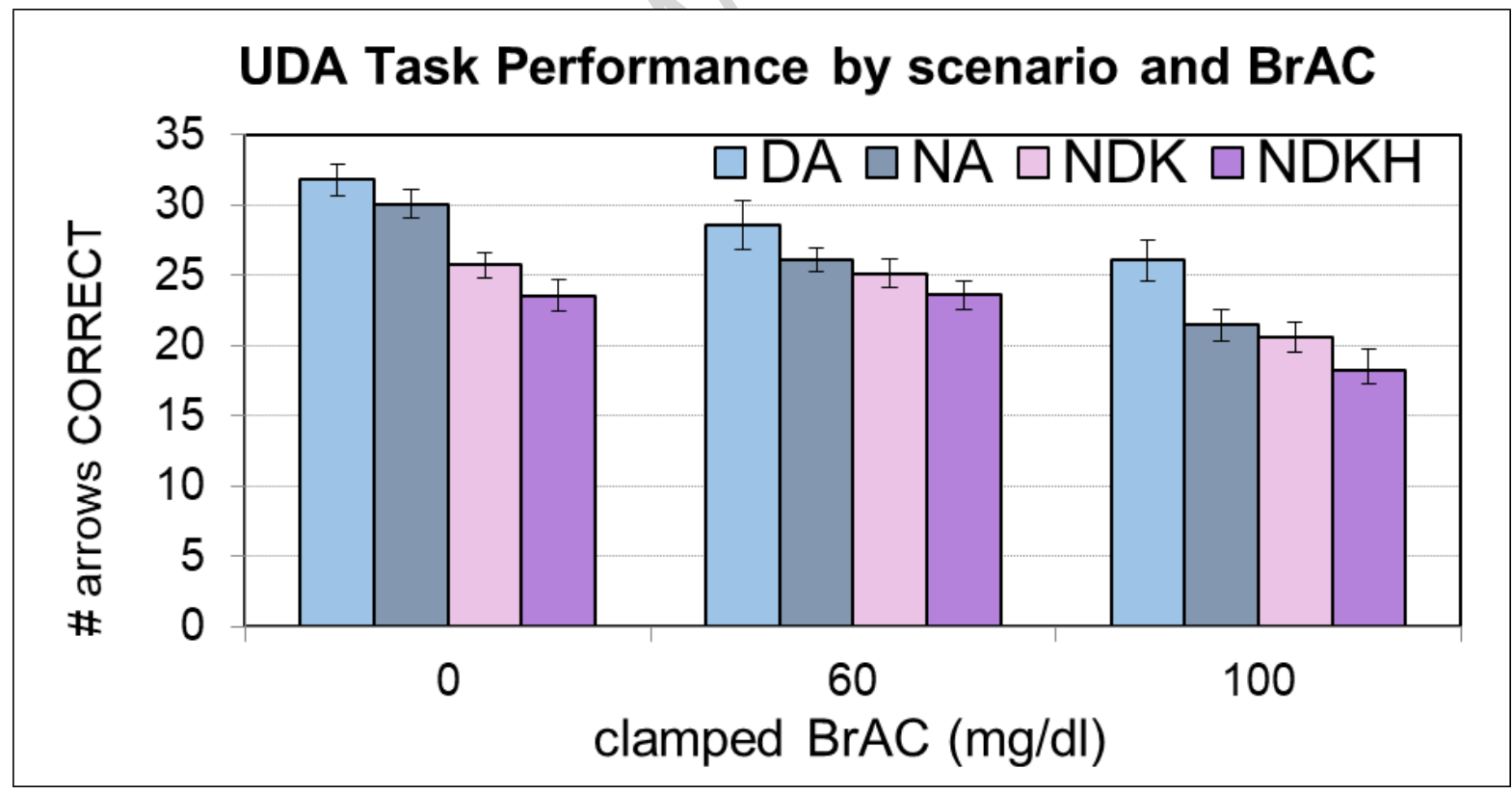

Figure 4: The number of environmentally embedded arrows correctly identified (mean \pm sem) for the 3 levels of clamped BrAC in each of the 4 driving scenarios in this study. Post hoc observation supported all of the project's hypotheses. 


\section{Discussion}

Our study found main effects of driving scenario and level of alcohol intoxication on a quantified measure of environmental vigilance in a sample of ten young adult healthy drinkers. We interpret the results as suggesting that alcohol impairs environmental vigilance in all conditions tested. We could not test an interaction between intoxication level and scenario difficulty for lack of a quantified measure of the latter. We believe we have demonstrated a significant alcohol exposure-relationship to the effect of intoxication on UDA vigilance, and that environmental vigilance is significantly degraded by alcohol intoxication at a $\mathrm{BrAC}$ below the current USA per se legal limit.

An alternative interpretation is that, in every scenario tested, alcohol progressively decreases the driver's total cognitive capacity which is already divided between driving the car and monitoring environmental cues relevant to driving safety.

The utility of combining the BrAC clamping method of exposing every driver to precisely the same BrAC level with a within-subject experimental design was apparent in this study. In regard to the independent variable, the standard deviations of BrAC during scenario performance were 5 to 7 times smaller than those reported in simulator studies using an oral route of alcohol administration (e.g. Tippin et al, 2009). Despite our small sample size, the reduced variance in the independent variable contribute to statistically and clinically significant observations that may have otherwise gone undetected using alcohol ingestion methods. Our within-subject experimental design minimized the potential influence of differences across subjects, such as native driving skills, driving experience, drinking histories, and gender, on performing the UDA task. The effect of such variability would be included in a between-group design and would likely require a larger sample size in order to test the effects of alcohol on environmental vigilance observed here. 
Vigilance tasks have been used in simulator studies of the effect of alcohol elsewhere (e.g. Ratcliff and Strayer, 2014, Jex et al, 1966), and alcohol intoxication was significantly associated with degradation in performance. However, to our knowledge, no study has embedded the stimuli in the environment during the performance of the driving scenario.

'Distraction' during driving is anathema to driving performance in conventional wisdom. Many credible simulator studies demonstrate this association, as well as a potent, even synergistic, effect of alcohol intoxication on driving performance while performing a distracting task (Van Dyke and Fillmore, 2015).

Is distraction the opposite of vigilance? Environmental vigilance is good for safety, surely, but may involve cognitive interference from the neural systems activity employed in controlling conventional measures of driving performance. Performing the UDA task requires a minimal motoric response (the movement of the right thumb within its normal range while using either the fingers of the right hand or both hands to steer the car), and the response required negligible visual effort. Detection of the UDA stimuli could be performed easily with only saccadic eye-movements, i.e. requiring no change in head position. Nonetheless, our results could be interpreted as if performing the UDA task was a distraction to driving the car, as would be consistent with the alcohol myopia theory (Sevincer and Oettingen, 2014). If taken in that context, we believe the UDA task quantifies the ability to perceive and interpret essential environmental distractions. We performed no scenarios without the concomitant UDA task embedded, so no assessment of the task, per se, as a distraction is available, but it would be interesting to know if successful vigilance required the same brain circuits (Xu et al, 2017) to be active during the other 96 percent of the driving time. Unfortunately, we cannot answer if distraction is the opposite of vigilance, but have found no clear answer in the literature, either.

Our study must be considered in light of its limitations. The main concern is that the fixed order of BrAC exposure, combined with testing 4 scenarios per exposure, invites an untested 
confound of our results with the effect of fatigue. The order of BrAC level, if tested in one session, is necessarily increasing because the natural clearance rate of alcohol from the body is slow (around $15 \mathrm{mg} / \mathrm{dl}$ per hour). The main issue here is the effect of any fatigue on driving performance attributable to the time subjects spent in the simulator; not the well-known effect of fatigue due to sleep deprivation (Arnedt JT et al., 2001); our subjects were well-rested and the testing occurred at mid-day. When asked, none of our subjects reported fatigue in the debriefing conducted an hour after the last scenario was performed, but alcohol may have masked that perception (Fairclough and Graham. 1999). We are, however, confident in the alcohol effects observed: such an effect was observed with the simplest scenario (DA) at the lowest non-zero BrAC level, corresponding to the smallest total amount of simulator time. A second simulator session for each subject, repeating all procedures with a non-alcoholic infusate, might have contributed insight into the fatigue issue, but budgetary resources were insufficient. Thus, our design and database do not support any rigorous testing for a fatigue effect. Another potential confounding issue is practice. In the latter regard, we perceive that, whatever effects practice and fatigue may have had on UDA performance, they are likely to have been in offsetting directions.

This study covered too many dimensions (task difficulty, BrAC level, driving performance and UDA task performance) for definitive statistical inference given our limited sample size, and separate formal replications of associations with multiple BrAC levels, but using one scenario per session (alternatively, one BrAC level and multiple scenarios) are probably required. Either alternative would require more than one session per subject in order to maintain the power advantage of within-subject design.

Nonetheless, we conclude that alcohol intoxication has an exposure-related, deleterious impact on drivers' capacity for environmental vigilance. 


\section{Acknowledgements}

Subject and pharmacy fees were paid by the Psychiatry Research Committee Study Program. Nursing support was provided by the Indiana Clinical Research Center. Investigator and technician salaries were covered by the Departments of Psychiatry, Neurology, and Electrical and Computer Engineering. The expense associated with programming and use of the driving simulator was donated by TASI. We gratefully acknowledge Jim Hays and James Millward for the proficient exercise of technician skills required for BrAC clamping, data reduction and database preparation. All authors contributed to the design, execution and manuscript preparation for this project; none have any intellectual or financial conflicts. 


\section{$\underline{\text { References }}$}

Arnedt JT, Wilde GJ, Munt PW, MacLean AW. (2001) How do prolonged wakefulness and alcohol compare in the decrements they produce on a simulated driving task? Accid Anal Prev. 33(3):337-44.

Bernosky-Smith KA, Shannon EE, Roth AJ, Liguori A (2011) Alcohol Effects on Simulated Driving in Frequent and Infrequent Binge Drinkers Hum Psychopharmacol. Hum Psychopharmacol. 26(3): 216-223. PMC3161131

Brooks JO., Goodenough RR., Crisler MC, Klein ND, Alley RL, Koon BL., Logan WC Jr, Ogle JH., Tyrrell RA., Wills RF. (2010) Simulator sickness during driving simulation studies. Accid Anal Prev. 42(3):788-96.

Fairclough SH, Graham R (1999) Impairment of driving performance caused by sleep deprivation or alcohol: a comparative study. Hum Factors. 41(1):118-28.

Fell JC, Voas RB (2014) The effectiveness of a 0.05 blood alcohol concentration (BAC) limit for driving in the United States Addiction. 109(6): 869-874.

Gomez R, Behar KL, WatzI J, Weinzimer SA, Gulanski B, Sanacora G, Koretski J, Guidone E, Jiang L, Petrakis IL, Pittman B, Krystal JH, Mason GF. (2012) Intravenous ethanol infusion decreases human cortical $\mathrm{Y}$-aminobutyric acid and $\mathrm{N}$-acetylaspartate as measured with proton magnetic resonance spectroscopy at 4 tesla. Biol Psychiatry. 71(3):239-46.

Laude JR, Fillmore MT (2015) Simulated driving performance under alcohol: effects on driverrisk versus driver-skill Drug Alcohol Depend. 1; 154: 271-277.

Kenntner-Mabiala R, Kaussner Y,Jagiellowicz-Kaufmannn M, Hoffmann MS, Krüger H-P (2015) Driving Performance Under Alcohol in Simulated Representative Driving Tasks: An Alcohol 
Calibration Study for Impairments Related to Medicinal Drugs J Clin Psychopharmacol. 35(2): 134-142. PMC4345970

Jex HR, McDonnell JD, Phatak AV (1966) A "critical" tracking task for man-machine research related to the operator's effective delay time. I. Theory and experiments with a first-order divergent controlled element. NASA CR-616. NASA Contract Rep NASA CR. Nov:1-105.

Jones AW, Norberg A, Hahn RG. (1997) Concentration-time profiles of ethanol in arterial and venous blood and end-expired breath during and after intravenous infusion. J Forensic Sci. 42(6):1088-94.

Meda SA, Calhoun VD, Astur RS, Turner BM, Ruopp K, Pearlson GD (2009) Alcohol Dose Effects on Brain Circuits During Simulated Driving: An fMRI Study Hum Brain Mapp. 30(4): 1257-1270. PMC2751645

National Transportation Safety Board, Bureau of Safety Programs, \& United States of America. (2013) Reaching Zero: Actions to Eliminate Alcohol-Impaired Driving

Plawecki MH, Decarlo R, Ramchandani VA, O'Connor S. (2007) Improved transformation of morphometric measurements for a priori parameter estimation in a physiologically-based pharmacokinetic model of ethanol. Biomed Signal Process Control. 2(2):97-110.

Plawecki M, Han JJ, Doerschuk P, Ramchandani V, O'Connor S (2008) Physiologically Based (PBPK) Models for Ethanol. IEEE Trans on BioMed. Eng 55:12 2691-2700

Rakauskas ME, Ward NJ, Boer ER, Bernat EM, Cadwallader M, Patrick CJ. (2008) Combined effects of alcohol and distraction on driving performance. Accid Anal Prev. 40:1742-1749

Ramchandani VA., Bolane .J, Li T-K, O'Connor S: (1999) A physiologically-based pharmacokinetic (PBPK) model for alcohol facilitates rapid BrAC clamping. Alcohol Cln Exp Res., 23:617-623 
Ramchandani VA., O'Connor, S. (2006) Studying Alcohol Elimination Using the Alcohol Clamp Method Alcohol Research \& Health Journal, 29(4): 286-291

Ratcliff R, Strayer S. (2014) Modeling Simple Driving Tasks with a One-Boundary Diffusion Model Psychon Bull Rev. 21(3): 577-589

Schweizer TA, Kan K, Hung Y, Tam F, Naglie G, Graham SJ (2013) Brain activity during driving with distraction: an immersive fMRI study. Front Hum Neurosci. 7():53.

Sevincer AT, Oettingen G. (2014) Alcohol myopia and goal commitment. Front Psychol. 4;5:169.

Sobell LC, Sobell MB (1992) Timeline Followback: A technique for assessing self-reported alcohol consumption. In: Litten RZ, Allen J, editors. Measuring alcohol consumption: Psychosocial and biological methods. New Jersey: Humana Press; pp. 41-72.

Strayer DL, Johnston WA (2001) Driven to distraction: dual-Task studies of simulated driving and conversing on a cellular telephone Psychol Sci. 12(6):462-6.

Tippin J, Sparks JD, Rizzo M (2009) Visual Vigilance in Drivers with Obstructive Sleep Apnea Psychosom Res. J Psychosom Res. 67(2): 143-151.

Van Dyke NA, Fillmore MT (2015) Distraction produces over-additive increases in the degree to which alcohol impairs driving performance; Psychopharmacology (Berl). 232(23): 42774284. PMC4618724

Veldstra JL, Bosker WM, de Waard D, Ramaekers JG, Brookhuis KA (2015) Comparing treatment effects of oral THC on simulated and on-the-road driving performance: testing the validity of driving simulator drug research Psychopharmacology (Berl) 232(16): 2911-2919 
Xu G, Zhang M, Wang Y, Liu Z, Huo C, Li Z, Huo M (2017) Functional connectivity analysis of distracted drivers based on the wavelet phase coherence of functional near-infrared spectroscopy signals PLoS One.12(11): e0188329. 


\section{Graphical Abstract for}

Alcohol intoxication progressively impairs drivers' capacity to detect important environmental stimuli; a pilot study.

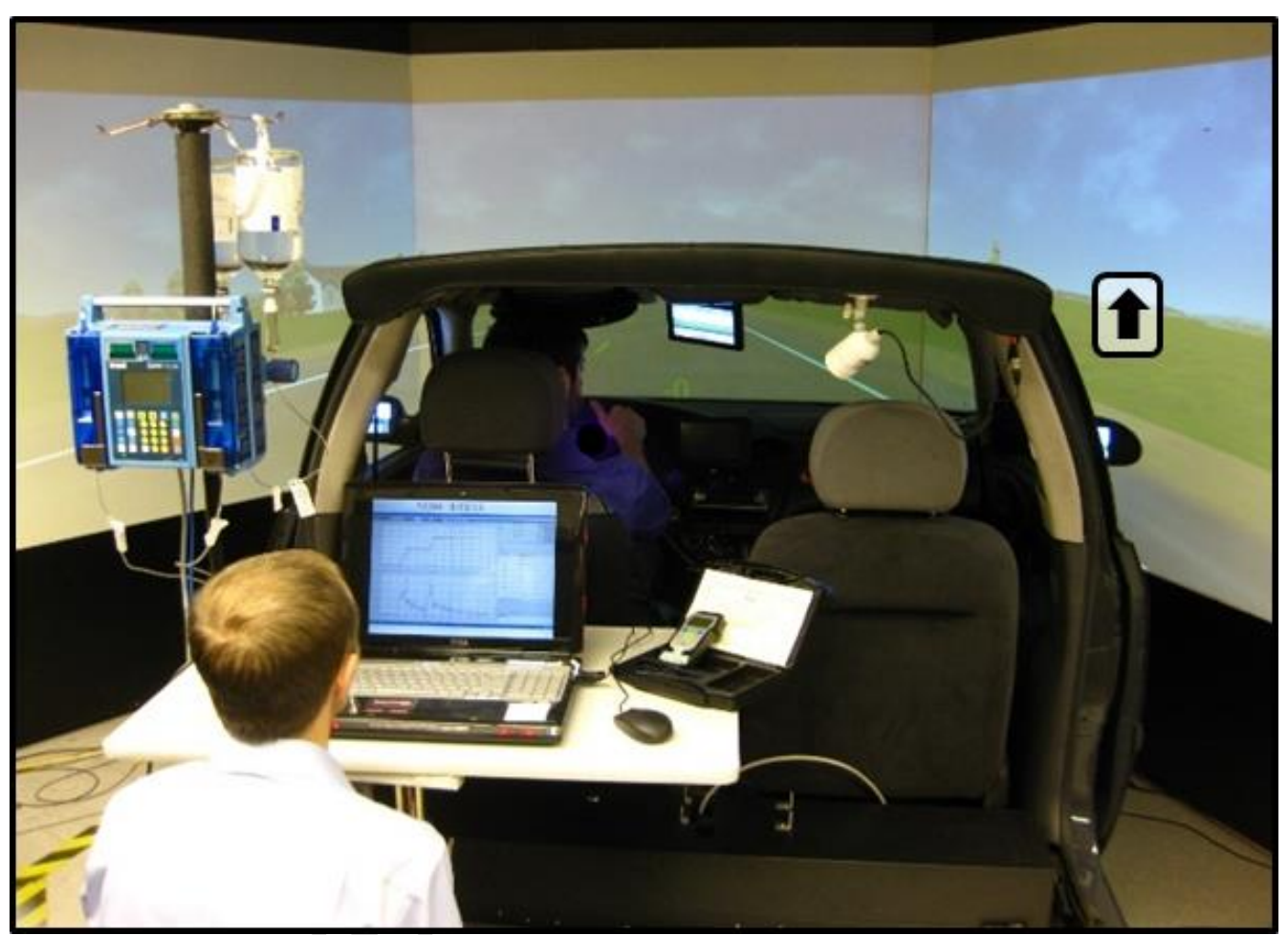

Authors:

Martin Henry Plawecki, M.D., Ph.D. ${ }^{\text {a1 }}$

Sarah Koskie, Ph.D. ${ }^{b}$

Ann Kosobud, Ph.D. ${ }^{\text {a2 }}$

Michael D. Justiss, Ph.D., OTR/L ${ }^{\mathrm{b}, \mathrm{c}}$

Sean O'Connor, M.D. ${ }^{\text {a1 }}$ 


\section{Highlights Page for}

\section{Alcohol intoxication progressively impairs drivers' capacity to detect important environmental stimuli; a pilot study.}

Corresponding Author:

Sean O'Connor, M.D.

Professor of Psychiatry and Biomedical Engineering

Indiana University School of Medicine and Purdue University

UH 5510 - General Clinical Research Center

500 University Boulevard

Indianapolis, IN, 46202 USA

(317) 989-4186 oconnor1@iu.edu

Highlights:

- Drivers need to remain vigilant for environmental cues related to driving safety.

- Alcohol intoxication reduces the driver's environmental vigilance.

- The impairment in vigilance is sensitive to driving conditions.

- Impairment of vigilance worsens with greater breath alcohol concentration. 